\title{
Familial nasopharyngeal carcinoma in Hong Kong: epidemiology and implication in screening
}

\author{
Wai Tong Ng · Cheuk Wai Choi • Michael C. H. Lee • \\ Siu Hong Chan · Tsz Kok Yau · Anne W. M. Lee
}

Published online: 10 May 2009

(C) Springer Science+Business Media B.V. 2009

\section{Erratum to: Familial Cancer (2009) 8:103-108 DOI 10.1007/s10689-008-9213-9}

Unfortunately the article published in the journal Familial Cancer (2009) 8:103-108 has following three corrections.

Due to a data transfer mistake for one patient, the authors regret that the following changes to be made.

The correct version of the eighth sentence in the abstract section must read as:

There were also more stage I-II patients in group 1 ( 38 vs $23 \%, P<0.01)$.

Instead of

There were also more stage I-II patients in group 1 (37 vs. $23 \%, P<0.01)$.
The correct version of the second sentence in the second paragraph of the Result section must read as:

Furthermore, more patients in group 1 presented in early stages than group 2, with $38 \%$ in group 1 presenting at stage I-II (stage I: $6.4 \%$, stage II: $31.2 \%$ ), compared to $23 \%$ (stage I: $0.6 \%$, stage II: $22.2 \%)$ in group $2(P<0.01)$.

Instead of

Furthermore, more patients in group 1 presented in early stages than group 2, with $37 \%$ in group 1 presenting at stage I-II (stage I: $6.4 \%$, stage II: $30.4 \%$ ), compared to $23 \%$ (stage I: $0.6 \%$, stage II: $22.2 \%)$ in group $2(P<0.01)$.

In Table 1, the second column under the "Screen detected (Group 1a)" is changed.

Please see the revised table shown below:

The online version of the original article can be found under doi:10.1007/s10689-008-9213-9.

W. T. Ng $(\bowtie)$ C. W. Choi · S. H. Chan .

T. K. Yau - A. W. M. Lee

Department of Clinical Oncology, Pamela Youde Nethersole

Eastern Hospital, 3 Lok Man Road, Chai Wan, Hong Kong

e-mail: ngwt1@ha.org.hk

M. C. H. Lee

Department of Medical Physics, Pamela Youde Nethersole

Eastern Hospital, 3 Lok Man Road, Chai Wan, Hong Kong 
Table 1 Patient characteristics

\begin{tabular}{|c|c|c|c|c|c|}
\hline & \multicolumn{2}{|c|}{ Group 1 (with family history) } & \multirow{2}{*}{$\begin{array}{l}\text { Group } 2 \text { (No } \\
\text { family history) }\end{array}$} & \multirow{2}{*}{$\begin{array}{l}P \text {-value (Group } 1 \\
\text { vs. Group 2) }\end{array}$} & \multirow{2}{*}{$\begin{array}{l}P \text {-value (Group 1b } \\
\text { vs. Group 2) }\end{array}$} \\
\hline & $\begin{array}{l}\text { Screen detected } \\
\text { (Group 1a) }\end{array}$ & $\begin{array}{l}\text { Symptomatic cases } \\
\text { (Group } 1 \mathrm{~b})\end{array}$ & & & \\
\hline Age & 41.8 & 48.7 & 50.3 & $0.044^{\mathrm{b}}$ & 0.293 \\
\hline Sex (male vs. female) & 41.2 vs. $58.8 \%$ & 66.7 vs. $33.3 \%$ & 74.8 vs. $25.2 \%$ & $0.005^{\mathrm{b}}$ & 0.065 \\
\hline $\begin{array}{l}\text { Duration of presenting } \\
\text { symptoms (month) }\end{array}$ & Not applicable & 4.74 & 5.30 & - & 0.882 \\
\hline $\mathrm{T}$ stage & & & & $0.039^{\mathrm{b}}$ & 0.427 \\
\hline $\mathrm{T} 1$ & $8(47.1 \%)$ & $2(1.9 \%)$ & $36(3.3 \%)$ & & \\
\hline $\mathrm{T} 2$ & $5(29.4 \%)$ & $55(50.9 \%)$ & $451(41.9 \%)$ & & \\
\hline $\mathrm{T} 3$ & $3(17.6 \%)$ & $26(24.1 \%)$ & $290(26.9 \%)$ & & \\
\hline $\mathrm{T} 4$ & $1(5.9 \%)$ & $20(18.5 \%)$ & $251(23.3 \%)$ & & \\
\hline Unknown $^{\mathrm{a}}$ & & $5(4.6 \%)$ & $49(4.5 \%)$ & & \\
\hline $\mathrm{N}$ stage & & & & $0.049^{\mathrm{b}}$ & 0.271 \\
\hline No & $7(41.2 \%)$ & $17(15.7 \%)$ & $132(12.3 \%)$ & & \\
\hline N1 & $6(35.3 \%)$ & $40(37.0 \%)$ & $317(29.4 \%)$ & & \\
\hline $\mathrm{N} 2$ & $4(23.5 \%)$ & $31(28.7 \%)$ & $395(36.7 \%)$ & & \\
\hline N3 & $0(0.0 \%)$ & $15(13.9 \%)$ & $184(17.1 \%)$ & & \\
\hline Unknown $^{\mathrm{a}}$ & & $5(4.6 \%)$ & $49(4.5 \%)$ & & \\
\hline M1 stage & $0(0.0 \%)$ & $5(4.6 \%)$ & $49(4.5 \%)$ & 0.779 & 0.970 \\
\hline Group stage & & & & $<0.001$ & 0.008 \\
\hline Early stage (I/II) & $10(58.8 \%)$ & $37(34.3 \%)$ & $246(22.8 \%)$ & & \\
\hline Advanced stage (III/IV) & $7(41.2 \%)$ & $71(65.7 \%)$ & $831(77.2 \%)$ & & \\
\hline
\end{tabular}

${ }^{a}$ Not assessed by imaging because of metastatic disease (i.e. IVC disease)

b Changed from significant to non-significant when compared with symptomatic cases only 